(c) American Dairy Science Association, 2006.

\title{
Short Communication: A Milk Trait-Associated Polymorphism in the Bovine Growth Hormone Receptor Gene Does Not Affect Receptor Signaling
}

\author{
Y. Zhou and $\mathrm{H}$. Jiang ${ }^{1}$ \\ Department of Animal and Poultry Sciences, Virginia Polytechnic Institute and State University, Blacksburg 24061
}

\begin{abstract}
Growth hormone (GH), also known as somatotropin, stimulates milk production in cows. At the tissue level, the action of $\mathrm{GH}$ is mediated by the $\mathrm{GH}$ receptor (GHR) and the receptor-activated intracellular signaling pathway involving Janus kinase 2 (JAK2) and signal transducer and activator of transcription 5 (STAT5). A T/A nucleotide variation in exon 8 of the bovine GHR gene, resulting in a phenylalanine to tyrosine change in the transmembrane domain of the GHR protein, has been reported to be associated with a major effect on milk yield in cows. The objective of this study was to determine whether the 2 versions of GHR differ in mediating GH-induced STAT5 activation of gene expression. We created cDNA expression plasmids for the 2 versions of GHR and cotransfected each of them with a STAT5 expression plasmid and a luciferase reporter gene construct containing STAT5 binding sites into 2 different cell lines. Treatment of the transfected cells with various concentrations of GH triggered a dose-dependent increase in luciferase activity. However, the GH-induced luciferase activity was not different between the 2 GHR expression plasmids, indicating that the 2 GHR forms did not differ in mediating GH-induced STAT5 activation of gene expression. Thus, if the T/A polymorphism in exon 8 of the GHR gene has a causative effect on milk production, this effect is unlikely to be mediated by the JAK2-STAT5 pathway, the currently known major signaling pathway from the growth hormone receptor.
\end{abstract}

Key words: single nucleotide polymorphism, growth hormone receptor, milk production

Growth hormone (GH), also known as somatotropin, is a major stimulator of postnatal growth and milk production in cattle (Etherton and Bauman, 1998). At the tissue level, the GH action is mediated by a specific cell membrane receptor, the growth hormone receptor

Received September 8, 2005.

Accepted December 12, 2005.

${ }^{1}$ Corresponding author: hojiang@vt.edu
(GHR). Binding of GH to GHR activates the Janus kinase 2 (JAK2); activated JAK2 in turn activates signal transducer and activator of transcription 5 (STAT5) through phosphorylation; phosphorylated STAT5 translocates from the cytoplasm to the nucleus, where it binds to specific DNA regions and activates transcription (Herrington and Carter-Su, 2001). A well-known gene controlled by GH through this JAK2-STAT5 pathway is IGF-I, which is believed to mediate most of the growth-stimulating and at least part of the milk production-stimulating effect of GH (Stewart and Rotwein, 1996; Etherton and Bauman, 1998). In addition to STAT5, GH-activated JAK2 also phosphorylates insulin receptor substrate 1, phospholipase C, and SHC protein, leading to changes in gene expression, enzymatic activity, or metabolite transport (Herrington and Carter-Su, 2001).

As predicted from its cDNA sequence (Hauser et al., 1990), the bovine GHR protein is a single-chain polypeptide of 634 amino acids, composed of an 18-AA signal peptide (not present in mature GHR protein) encoded by exon 2 of the GHR gene, a 242-AA extracellular domain encoded by exons 3 to 7, a 24-AA single transmembrane domain encoded by exon 8 , and a 350-AA intracellular domain encoded by exons 9 and 10 . The bovine GHR mRNA is heterogeneous in the 5 '-untranslated region, due to initiation of transcription from different leader exons (or alternative exon 1) and alternative splicing (Jiang and Lucy, 2001). Because the action of GH depends on GHR, genetic variations in the GHR gene might change the GHR amino acid sequence or expression level, thereby affecting GH-controlled traits such as growth and milk production in animals. Through QTL mapping, Blott et al. (2003) found that a T/A single nucleotide variation in exon 8 of the bovine GHR gene [the corresponding 2 GHR DNA or cDNA forms are designated GHR-E8(T) and GHR-E8(A)] results in a phenylalanine ( $\mathrm{F}$ ) to tyrosine $(\mathrm{Y})$ change at the 279th residue, a residue of the transmembrane domain (Figure 1), of the GHR protein. The corresponding GHR protein forms are designated GHR279F and GHR279Y, respectively, and are associated with a major effect on milk yield in Ayrshire, Holstein, and Jersey cows. Cows 


\section{GHR-E8(T)}
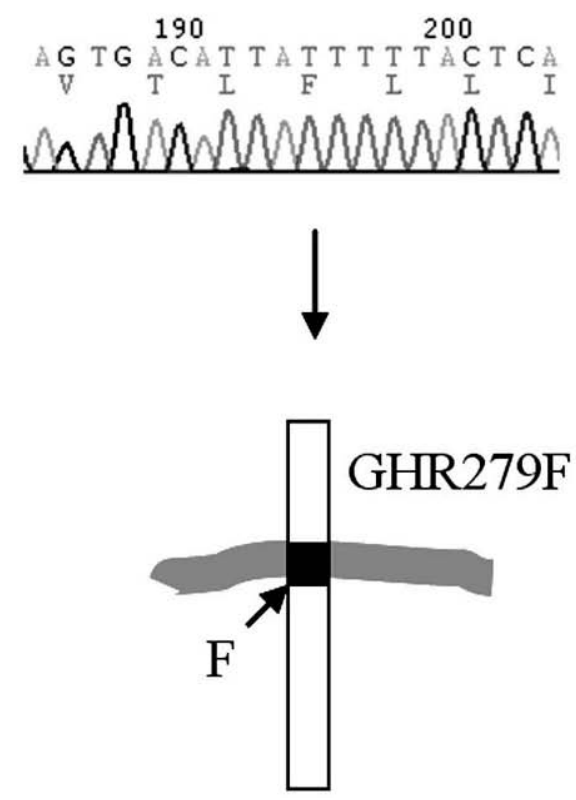

GHR-E8(A)
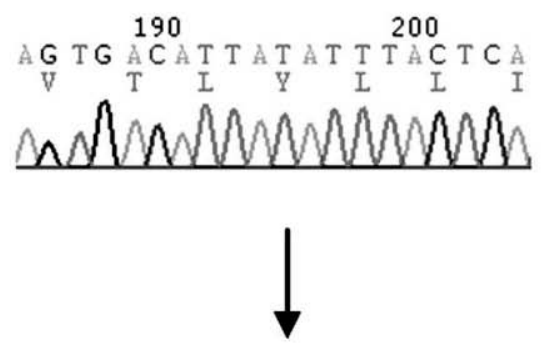

membrane

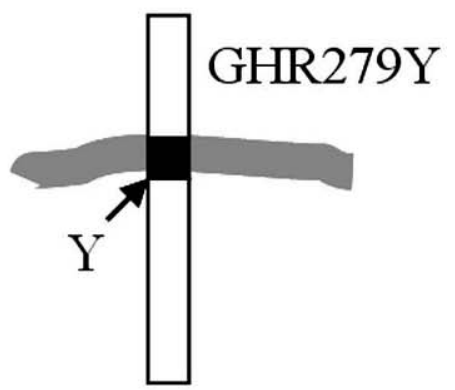

Figure 1. Schematic representation of the milk trait-associated single nucleotide polymorphism in exon 8 (E8) of the bovine growth hormone receptor (GHR) gene and the corresponding changes in the GHR protein. The T to A nucleotide variation in exon 8 of the bovine GHR gene [GHR-E8(T) or GHR-E8(A)] results in a phenylalanine (F) to tyrosine (Y) change at the 279th residue (a residue located within the transmembrane domain) of the receptor.

with GHR279Y produce about $200 \mathrm{~kg}$ more milk annually than the cows carrying GHR279F (Blott et al., 2003). The same authors also indicated that the single nucleotide polymorphism had a similar effect on live weight in cattle (Blott et al., 2003). Given the wellknown effect of $\mathrm{GH}$ on milk production in cows, an obvious hypothesis for why GHR279Y is associated with greater milk yield than GHR279F is that GHR279Y mediates greater $\mathrm{GH}$ action than GHR279F. In this study we compared the ability of GHR279Y and GHR279F to mediate GH-induced STAT5 activation of gene expression in cell culture.

In a previous study (Wang and Jiang, 2005), we cloned, from a Holstein cow, a 2,026-bp bovine GHR cDNA (GenBank accession number AY748827) containing the entire open reading frame for the 634-AA GHR protein into the expression vector pcDNA3.1 (Invitrogen, Carlsbad, CA) between the EcoR V and Xba I restriction sites. The nucleotide at the polymorphic position of exon 8 in this GHR cDNA was T (nucleotide 914 in GenBank accession number AY748827); for the purpose of this study, this expression plasmid was renamed bGHR-E8(T)/pcDNA3. We mutated $\mathrm{T}$ to $\mathrm{A}$ at the polymorphic position in bGHR-E8(T)/pcDNA3, generating bGHR-E8(A)/pcDNA3. The mutagenesis was done by 3 rounds of PCR, using a mixture (10:1) of Taq DNA polymerase and high-fidelity DNA polymerase $T l i$
(Promega, Madison, WI). The first PCR amplified the GHR cDNA region 1 to 924 (in GenBank accession number AY748827), using forward primer 5'-GCCATAAA GCCTGGAGGAAC-3' and reverse primer 5'-TATGAG TAAATATAATGTCACTGCTAGC-3', where the nucleotide in boldface indicates the incorporated mutation; the second PCR amplified the GHR cDNA region 903 to 2,026 (in GenBank accession number AY748827), using forward primer 5'-AGTGACATTATATTTACTC ATATTTTC-3' (the nucleotide in boldface was the incorporated mutation) and reverse primer $5^{\prime}$-TCTCTCTA GACCTGTGCCATTCAATGGGTAG-3', where the sequence underlined is an $\mathrm{Xba}$ I restriction site. The products of these 2 PCR were mixed and from this mixture, the 2,026-bp GHR-E8(A) cDNA was amplified by the third PCR, using the same forward primer as used in the first PCR and the same reverse primer as used in the second PCR. The product of the third PCR was cloned into pcDNA3.1 between the $E c o \mathrm{R} \mathrm{V}$ and $X b a \mathrm{I}$ sites, generating bGHR-E8(A)/pcDNA3. The GHR cDNA insert in this plasmid was sequenced completely to confirm that it differed from GHR-E8(T) cDNA only at the polymorphic nucleotide position of exon 8 (Figure 1).

We then cotransfected $0.2 \mu \mathrm{g}$ of bGHR-E8(T)/pcDNA3 or bGHR-E8(A)/pcDNA3 or pcDNA3.1 (no-GHR control), $0.2 \mu \mathrm{g}$ of a STAT5b expression plasmid (Ariyoshi 

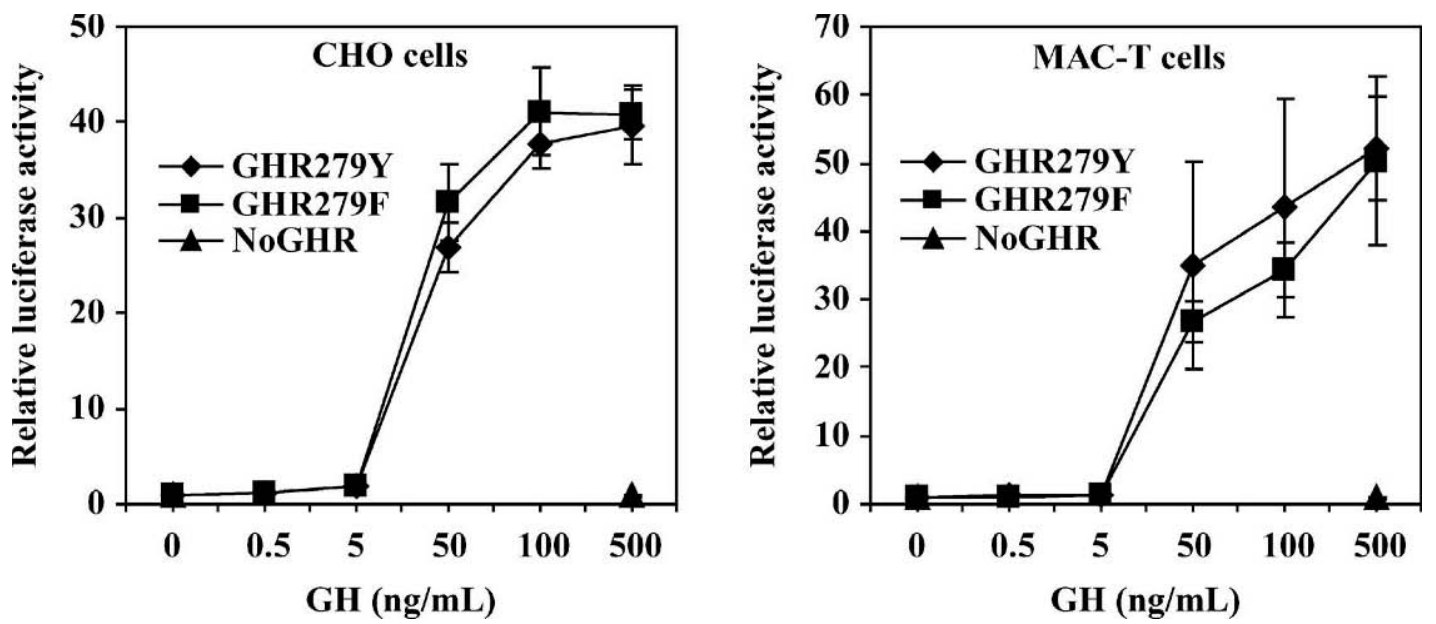

Figure 2. Comparison of the ability of the bovine growth hormone receptor (GHR) containing phenylalanine (F) at the 279th residue (GHR279F) and the receptor containing tyrosine (Y) at the 279th residue (GHR279Y) to mediate growth hormone-induced STAT5 activation of reporter gene expression. A Chinese hamster ovary cell line (CHO) and bovine mammary epithelial cell line (MAC-T) were transfected with a STAT5b expression plasmid, a STAT5 binding site-containing reporter gene plasmid, a transfection efficiency control plasmid, and a GHR279F-encoding plasmid (GHR279F) or a GHR279Y-encoding plasmid (GHR279Y) or the pcDNA3.1 plasmid that did not contain GHR cDNA insert (no GHR). The transfected cells were treated with indicated concentrations of recombinant bovine GH for $8 \mathrm{~h}$ (the no-GHR cells were treated with $500 \mathrm{ng} / \mathrm{mL}$ of $\mathrm{GH}$ only), followed by dual-luciferase assay. Values are expressed as mean \pm standard error of the mean. Growth hormone caused a dose-dependent activation of luciferase reporter gene expression in both cell types, and activation depended on inclusion of the GHR expression plasmid in the transfection. Within each cell type, GH-induced luciferase activity was not different $(P$ $>0.05$ ) between GHR279F and GHR279Y.

et al., 2000), $0.2 \mu \mathrm{g}$ of pSPI-LUC, a firefly luciferase reporter plasmid that contained a growth hormone response element constituted by 6 copies of STAT5 binding site from the Spi 2.1 gene (Wood et al., 1997), and $0.5 \mathrm{ng}$ of pRL-CMV, a Renilla luciferase-encoding plasmid for transfection efficiency control, into a Chinese hamster ovary-derived cell line (CHO, American Tissue Culture Collection, Manassas, VA) and a bovine mammary epithelia-derived cell line (MAC-T, Huynh et al., 1991), using FuGENE6 (Roche, Indianapolis, IN) as transfection reagent. Both $\mathrm{CHO}$ and MAC-T cells were cultured in 24-well plates in Dulbecco's modified Eagle's medium supplemented with $10 \%$ fetal bovine serum at $37^{\circ} \mathrm{C}$ under $5 \% \mathrm{CO}_{2}$. In both cell lines, endogenous GHR expression was undetectable (Emtner et al., 1990; H. Jiang, Y. Zhou, and Y. Wang, Virginia Polytechnic Institute and State University, Blacksburg, unpublished data). Twenty-four hours after transfection, the medium was replaced with serum-free Dulbecco's modified Eagle's medium, and the culture was continued for 16 $\mathrm{h}$. The transfected cells were subsequently treated with different concentrations of recombinant bovine $\mathrm{GH}$ (provided by A. F. Parlow, National Hormone and Peptide Program, Torrance, CA) or PBS, the vehicle for $\mathrm{GH}$, for $8 \mathrm{~h}$ before being lysed for dual-luciferase assay (Promega). This transfection analysis was repeated 4 times in $\mathrm{CHO}$ cells and 3 times in MAC-T cells. The luciferase activity expressed from the pSPI-LUC construct was divided by that from pRL-CMV in the same well to normalize well-to-well variation in transfection efficiency. The adjusted luciferase activity data for the same GHR construct were compared between different concentrations of GH by one-factor ANOVA, followed by the Tukey's procedure. The adjusted luciferase activity data for the 2 different GHR constructs at each GH concentration were compared by the $t$-test. These statistical analyses were done using the respective programs of SAS (SAS Institute, Inc., Cary, NC). Differences at $P<0.05$ were considered significant.

As shown in Figure 2, GH caused a dose-dependent increase in luciferase activity, with significant induction $(P<0.05)$ starting to occur at $5 \mathrm{ng} / \mathrm{mL}$ in both $\mathrm{CHO}$ and MAC-T cells. This response was dependent on the GHR cDNA plasmid, because GH treatment failed to induce luciferase expression when the GHR expression plasmid was omitted in the transfection (Figure 2). The CHO or MAC-T cells transfected with bGHR-E8(T)/ pcDNA3 and the same type of cells transfected with bGHR-E8(A)/pcDNA3 expressed similar levels $(P>$ 0.05 ) of luciferase activity in response to various $\mathrm{GH}$ concentrations (Figure 2), indicating that the 2 versions of GHR, GHR279Y and GHR279F (expected to be expressed from bGHR-E8(A)/pcDNA3 and bGHR-E8(T)/ pcDNA3, respectively) did not differ in their ability to mediate GH-induced STAT5 activation of reporter gene expression in $\mathrm{CHO}$ or MAC-T cells.

That GHR279Y and GHR279F do not differ in mediating GH activation of JAK2-STAT5 signaling is per- 
haps not surprising, because the 279th residue of the receptor is located in the transmembrane domain (Figure 1), a region that is not involved in association with JAK2 or any other known intracellular signaling components (Argetsinger and Carter-Su, 1996). Hence, if the T/A nucleotide polymorphism in exon 8 of the GHR gene has a causative effect on milk production in cows, this effect is unlikely to be mediated by the JAK2STAT5 signaling pathway, the currently known major signaling pathway from the growth hormone receptor (Herrington and Carter-Su, 2001).

\section{ACKNOWLEDGMENTS}

The authors thank Kouichi Ariyoshi (University of Tokyo) and Tim Wood (Karolinska Institute) for providing the STAT5 and pSPI-LUC plasmids, respectively.

\section{REFERENCES}

Argetsinger, L. S., and C. Carter-Su. 1996. Mechanism of signaling by growth hormone receptor. Physiol. Rev. 76:1089-1107.

Ariyoshi, K., T. Nosaka, K. Yamada, M. Onishi, Y. Oka, A. Miyajima, and T. Kitamura. 2000. Constitutive activation of STAT5 by a point mutation in the SH2 domain. J. Biol. Chem. 275:2440724413.

Blott, S., J. J. Kim, S. Moisio, A. Schmidt-Kuntzel, A. Cornet, P. Berzi, N. Cambisano, C. Ford, B. Grisart, D. Johnson, L. Karim, P. Simon, R. Snell, R. Spelman, J. Wong, J. Vilkki, M. Georges,
F. Farnir, and W. Coppieters. 2003. Molecular dissection of a quantitative trait locus: A phenylalanine-to-tyrosine substitution in the transmembrane domain of the bovine growth hormone receptor is associated with a major effect on milk yield and composition. Genetics 163:253-266.

Emtner, M., L. S. Mathews, and G. Norstedt. 1990. Growth hormone (GH) stimulates protein synthesis in cells transfected with GH receptor complementary DNA. Mol. Endocrinol. 4:2014-2020.

Etherton, T. D., and D. E. Bauman. 1998. Biology of somatotropin in growth and lactation of domestic animals. Physiol. Rev. 78:745-761.

Hauser, S. D., M. F. McGrath, R. J. Collier, and G. G. Krivi. 1990. Cloning and in vivo expression of bovine growth hormone receptor mRNA. Mol. Cell. Endocrinol. 72:187-200.

Herrington, J., and C. Carter-Su. 2001. Signaling pathways activated by the growth hormone receptor. Trends Endocrinol. Metab. 12:252-257.

Huynh, H. T., G. Robitaille, and J. D. Turner. 1991. Establishment of bovine mammary epithelial cells (MAC-T): An in vitro model for bovine lactation. Exp. Cell Res. 197:191-199.

Jiang, H., and M. C. Lucy. 2001. Variants of the $5^{\prime}$-untranslated region of the bovine growth hormone receptor mRNA: Isolation, expression and effects on translational efficiency. Gene 265:4553.

Stewart, C. E., and P. Rotwein. 1996. Growth, differentiation, and survival: Multiple physiological functions for insulin-like growth factors. Physiol. Rev. 76:1005-1026.

Wang, Y., and H. Jiang. 2005. Identification of a distal STAT5-binding DNA region that may mediate growth hormone regulation of insulin-like growth factor-I gene expression. J. Biol. Chem. 280:10955-10963.

Wood, T. J., D. Sliva, P. E. Lobie, F. Goullieux, A. L. Mui, B. Groner, G. Norstedt, and L. A. Haldosen. 1997. Specificity of transcription enhancement via the STAT responsive element in the serine protease inhibitor 2.1 promoter. Mol. Cell. Endocrinol. 130:69-81. 